\title{
UV-BIOmarker Mapper Raman optical instrument for Venus atmosphere (UV-BIOMAP)
}

P. Ribes-Pleguezuelo, A. Sansano-Caramazana, H. Strese, L. Maresi

P. Ribes-Pleguezuelo, A. Sansano-Caramazana, H. Strese, L. Maresi, "UVBIOmarker Mapper Raman optical instrument for Venus atmosphere (UVBIOMAP)," Proc. SPIE 11852, International Conference on Space Optics ICSO 2020, 118523X (11 June 2021); doi: 10.1117/12.2599627

SPIE Event: International Conference on Space Optics - ICSO 2021, 2021, Online Only 


\section{International Conference on Space Optics-ICSO 2020}

Virtual Conference

30 March-2 April 2021

Edited by Bruno Cugny, Zoran Sodnik, and Nikos Karafolas
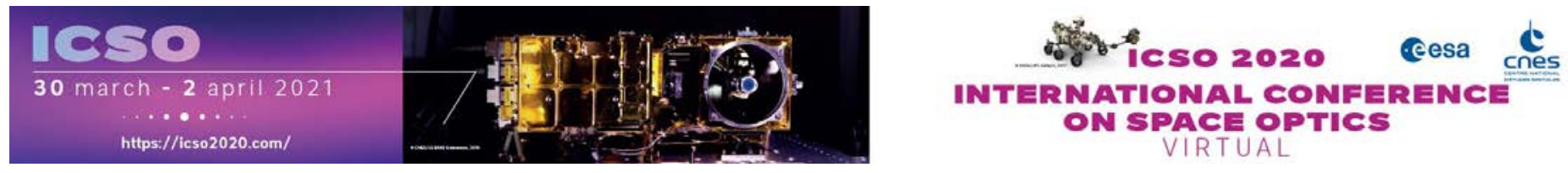

\section{UV-BIOmarker Mapper Raman optical instrument for Venus atmosphere (UV-BIOMAP)}

\section{Cesa issopocesalings lecnes}




\title{
UV-BIOmarker Mapper Raman optical instrument for Venus AtmosPhere (UV-BIOMAP)
}

\author{
P. Ribes-Pleguezuelo*a , A. Sansano-Caramazana ${ }^{\text {b,c }}$, H. Strese ${ }^{\mathrm{a}}$, L. Maresia \\ aEuropean Space Agency-European Space Research and Technology Centre, Keplerlaan 1, PO Box \\ 299 NL-2200 AG Noordwijk, The Netherlands; ${ }^{b}$ Trekroner Tecnologías y Proyectos SLU. \\ C/.Tamarindo 1, 2D E-47008 Valladolid, Spain; 'University of Valladolid. C/Plaza de Santa Cruz, 8, \\ 47002 Valladolid, Spain
}

\begin{abstract}
Several chemicals which could be relevant for life processes are found in the upper atmosphere of Venus $\left(\mathrm{H}_{2} \mathrm{~S}\right.$, OCS, $\mathrm{SO}_{2}, \mathrm{NH}_{4} \mathrm{Cl}, \mathrm{NH}_{2} \mathrm{COOHN} 4$ and $\mathrm{H}_{2} \mathrm{O}$ ). Moreover, the atmosphere instability and the reason for the high ultraviolet (UV) heterogeneous absorption between 320 and $400 \mathrm{~nm}$ in the top cloud layer and mesosphere $(\sim 40-70 \mathrm{Km})$ are still disputed phenomena. These scientific points could be further studied by small UV Raman and fluorescence instruments mounted in a small lander platform $(<20 \mathrm{~kg})$ being send to Venus.

In this current article we will propose an optical payload mission, which could be useful to give more information on these scientific questions. This study proposes a feasibility analysis for launching a small probe device that includes an emitting laser, a receiving telescope and a detector. The goal of this theoretical mission (UV-BIOmarker Mapper for Venus AtmosPhere or UV-BIOMAP) will be to analyse the laser-emitted and further absorption of UV light in the Venus atmosphere, and to verify the possible presence of biomarkers. The current state of the art of the required technologies to be implemented would allow a more cost-efficient and easy to develop mission, compared with previously completed Venus probes. This article will be focused on the required science and optical payload devices needed for solving these issues. Requirements for mass and power budgets for the optical payload will be analysed to ensure that the mission can be carried out at relatively low cost while still accomplishing the scientific goals of revealing the composition of Venus's atmosphere in more detail and the reason for the heterogeneous UV absorption in the planet's top cloud layer and mesosphere.
\end{abstract}

Keywords: Venus atmosphere, Raman, spectroscopy, ultraviolet, amino acid, fluorescence

\section{INTRODUCTION}

\subsection{Venus mission background}

Several chemical components that could represent the first steps for further organic processes are found in the upper atmosphere of Venus $\left(\mathrm{H}_{2} \mathrm{~S}, \mathrm{OCS}, \mathrm{SO}_{2}, \mathrm{NH}_{4} \mathrm{Cl}, \mathrm{NH}_{2} \mathrm{COOHN}_{4} \text { and } \mathrm{H}_{2} \mathrm{O}\right)^{1,2}$. These chemical components could easily lead to consecutive creation of amino acids ${ }^{1}$, which are the very first steps for further biological processes. As reported in ${ }^{3}$, UV Raman devices could be a key tool to detect the presence of such components. On the other hand, the high heterogeneous absorption between 320 and $400 \mathrm{~nm}$, in the top cloud layer and mesosphere $(\sim 40-70 \mathrm{Km})^{4}$ and the atmosphere disequilibrium, are still questions that could be potentially solved by using a single optical payload. The two main reasons to explain these processes could be the concentration of $\mathrm{FeCl}_{3}$ and $\mathrm{SiO}_{2}$, or the presence of non-oxygenic photosynthesis lifeforms ${ }^{5}$.

*pol.ribes@esa.int; phone+31 715655061 
An in-situ analysis tool, as proposed here, could be implemented by using a Raman spectroscopy device and a laserinduced florescence (LIF) device mounted on a landing probe. This study will help the further understanding of the composition and behaviour of the planet's atmosphere. Missions proposing the need for performing spectroscopy experiments on Venus clouds have already been suggested ${ }^{5}$. Examples of the most recent ones are the Venera-D mission from Russia ${ }^{6}$, which is already planning a probe instrument including an imaging camera, a chromato-mass spectrometer, an alpha P-X-spectrometer, a gamma spectrometer, a laser spectrometer, a hazemeter and a seismic detector $^{7}$. The spectrometer will use a multi-channel tuneable diode laser spectrometer. On the other hand, NASA started developing ideas for a CubeSat mission and descending probes to study Venus' atmosphere that incorporate similar instruments ${ }^{8,9}$.

The goal of our feasibility study will be to identify the specific science to be conducted, to propose an optical payload baseline design for a descending probe and to present a rough estimation of the budget for the mission realisation.

\subsection{Brief history of scientific missions in the Venusian atmosphere}

The history of Venus explorations starts in the early 60s with the first Russian missions performing flybys and landing on our nearest planet. Although these first missions were not as successful as planned, they paved the way for future Soviet missions which could finally analyse the planet's atmosphere. The first successful atmospheric mission, the Venera 4 probe $\left(383 \mathrm{Kg}\right.$ ), was carried out by the Soviet bloc in the year $1967^{10}$. The mission already included a UV spectrometer that helped to analyse the atmosphere composition of Venus for the first time. In the year 1984, the VeGa missions were launched from Baikonur. They successfully tested the possibility of studying the alien atmosphere by sending two landing probes installed in independent balloons deployed at around $60 \mathrm{~km}$ altitude ${ }^{11}$. The inclusion of a helium balloon to be deployed in the descending probe helped scientists to obtain a much longer analysis time and area of study, due to the dense atmosphere composition and presence of strong winds ${ }^{12}$. Earlier, in 1978, NASA also sent the Pioneer Venus multiprobe (around $600 \mathrm{Kg}$ ) that performed atmosphere analysis thanks to a battery of different independent probes and instruments (amongst others, a mass spectrometer and gas chromatograph to measure atmosphere composition, a cloud particle size spectrometer and temperature and pressure sensors) ${ }^{13}$.

From the 80 s on, many more missions have been sent to Venus; mainly by American, European, and more recently Japanese contributions. However, since the earliest first missions during the 60's to 80's decades, no more landing probes were sent into the planet's atmosphere. However, nowadays, the current advances in Raman spectrometers would be able to provide a much more accurate and extensive analysis than the missions four decades ago. Moreover, new technological developments would allow to do it on a much smaller and lighter platform, which would result in a much more cost-effective mission.

\subsection{Space Raman Devices}

Raman spectroscopy is an interesting technology for planetary science, since it allows for in situ analysis of samples thanks to the inelastic scattering of light. Fundamentally, the experiment just requires a narrow light excitation source, usually provided by a laser; and a spectrometer instrument to analyse the Raman light shift, which will provide accurate information of vibrational and rotational molecule levels of the illuminated sample. Raman spectroscopy can measure samples up to $200 \mathrm{~m}$ away and so can be useful for long-range atmospheric measurements ${ }^{3}$.

Several research groups have been working in the last decades to provide compact Raman systems that are capable of operating in extreme environments, including the possibility of operating in the different bodies of our Solar system. Already in 2006, the University of Hawaii implemented several changes to standard Raman spectroscopy devices to be able to create a small portable system to be operated outdoors ${ }^{14}$. The group, already planning for space exploration, designed a device using a $532 \mathrm{~nm}$ laser with an energy of $35 \mathrm{~mJ}$ and with $8 \mathrm{~ns}$ pulses. The team reported that they were successful in their goals, thanks to the use of volume holographic transmission gratings, which provided a better resolution (70-2470 $\mathrm{cm}^{-1}$ line spacing with a resolution of $\left.9 \mathrm{~cm}^{-1}\right)$. The device had a laser source using a Nd:YAG laser with second harmonic generation (SHG) to obtain the $532 \mathrm{~nm}$ emission. The incoming light from the sample was later collected thanks to a Makusutov Cassegrain $127 \mathrm{~mm}$ telescope ${ }^{14}$.

Furthermore, several planetary mission are currently using compact Raman devices to perform research. The best known example is the NASA 2020 mission to Mars. The mission will use the SuperCam instrument, to perform $7 \mathrm{~m}$ distance Raman analysis. The SuperCam includes, in addition to Raman, a suite of measurement techniques, such as LIBS, TimeResolved Fluorescence (TRF) spectroscopy, Visible and InfraRed (VISIR) reflectance spectroscopy and high-resolution 
imaging. The mission also uses a robotic arm that integrates the Scanning Habitable Environments with Raman \& Luminescence for Organics \& Chemicals (SHERLOC) Raman device, to perform proximity measurements. In this last case, the Raman device operates with a $248.6 \mathrm{~nm}$ laser emission ${ }^{15}$. Similarly, the European Space Agency (ESA) is planning to launch an instrument in the frame of the ExoMars mission in 2022, which will also have Raman capabilities $^{16,17}$.

\subsection{Venus atmosphere conditions}

One constraint of doing Raman spectroscopy in Venus's atmosphere, is the presence of extreme and challenging conditions under which the devices will have to perform. The investigation of existing chemicals and possible amino acids will have to be performed in very different pressure and temperature regimes, depending on the atmospheric altitude $^{18,19}$.

The atmospheric region of interest for the present study (although the probe could be analysing data down to the planet's surface) is the one between 70 to $40 \mathrm{~km}$ altitudes. At these altitudes, the atmosphere has a sulphuric acid clouded region, with temperatures approximately between 200 to $400 \mathrm{~K}$, and pressures approximately between 0.1 to $10 \mathrm{~atm}^{19}$.

In contrast, the presence of strong winds, up to $400 \mathrm{Km} / \mathrm{h}$, could make balloon exploration very favourable, since the probe would be able to travel horizontally, covering vast atmospheric areas ${ }^{20}$. This has already been used by the Russian VeGA mission in 1984, giving successful results, and was proposed also to ESA in 2013 in response to the Cosmic Vision Call for Ideas ${ }^{21}$. Once, and if the probes reach the planet's surface and are still analysing data, the instruments will encounter high pressures (around $90 \mathrm{~atm}$ ) and high temperatures (around $700 \mathrm{~K}$ ).

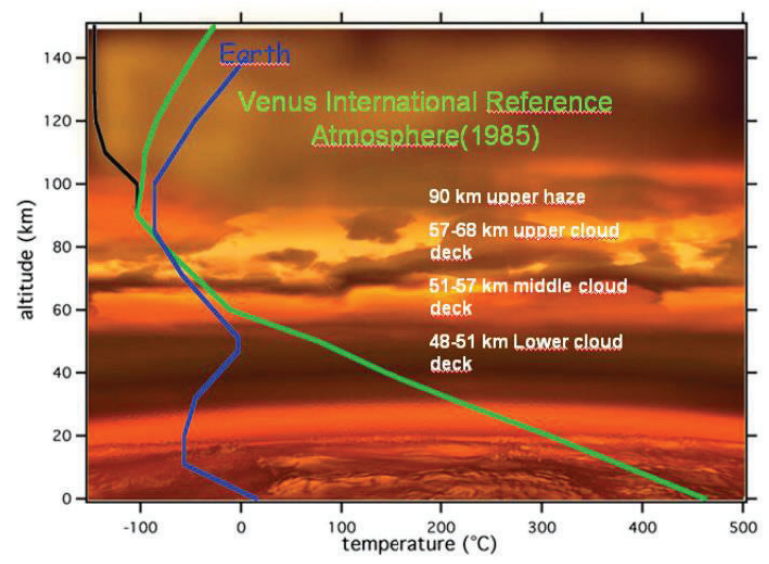

Figure 1 Venus atmospheric profile. Source ${ }^{22}$.

\section{PROPOSED MISSION GOALS AND REQUIRED DEVICES}

Our purpose is to show the possibility of developing a lander/planner probe device that includes an emitting laser, a receiving telescope and a spectrometer device. The goal of the mission will be to analyse the emission and absorption of UV light in Venus's atmosphere, and the possible presence of organic compounds. The current state-of-the-art of the required technologies allow for a moderate-cost and easy-to-develop mission ${ }^{20}$, compared with previously completed Venus probes.

The feasibility of the mission takes into consideration the possibility of being part of a bigger, already planned, Venusian mission, with enough space to include a small platform $(<20 \mathrm{Kg})$. The main reason for this is to be able to have a moderate-cost by avoiding those involved with launching and the transportation of the probe, plus using an existing mission communication system, to transfer the analysed data to an orbiter platform linked to Earth. This would help to decrease the required probe mass and power budgets. 


\subsection{Science}

Several scientific articles have theorized about the possibility of Venusian life in the planet's atmosphere ${ }^{5,23}$. Venus's atmosphere is mainly composed of $\mathrm{CO}_{2}$, but also contains other relevant elements related to Earthly biochemical processes. Moreover, the already mentioned UV absorption processes could result from a possible anoxygenic photosynthetic life form ${ }^{23}$.

Raman spectroscopy is a powerful analysis technique by which a precise identification of both mineral and organic phases can be carried out, without disturbing the analysed sample. This analysis can be done in any state in which the sample is found (solid, liquid or gas) and with micrometre resolution. In addition, Raman spectroscopy is a very fast technique that allows measurement in a few seconds. Along with this, the data products are very simple, so the memory consumption and processing are minimal. This technique can be applied both in contact mode, bringing the signal through optics and fibers to the spectrometer, as well as remotely, from a few centimetres to tens of meters. Contact measurements can be carried out using a continuous laser and in low light conditions, while remote acquisition is carried out using a pulsed laser, which allows samples to be analysed without interference from outside light and other phenomena which could interfere. Therefore, a properly designed instrument based on this technique can perform fast and detailed analysis of samples in-situ.

These characteristics and this design would make it very suitable for the detection of amino acids and their precursors in the atmosphere of Venus. There is already extensive experience in the use of this technology in the determination and differentiation of amino acids in various environments, whether deposited on inorganic surfaces, in solution or in aerosols. In addition, the Raman spectroscopy technique makes it possible to uniquely distinguish each type of amino acid by having a unique fingerprint based on its molecular composition. Moreover, in recently published articles ${ }^{24}$, it is stated that the discovery of phosphine in Venus's atmosphere could be produced by certain types of bacteria. This could also be easily studied by a Raman technique. In particular, researchers believe that performing distance Raman using a pulsed UV excitation source could help to determine the presence of organics, amino acids, or other more complex biomarker molecules. The Raman shift range between 500 and $1700 \mathrm{~cm}^{-1}$ gives a sufficient signal for the precise determination of the compounds. Which will further simplify the detection design.

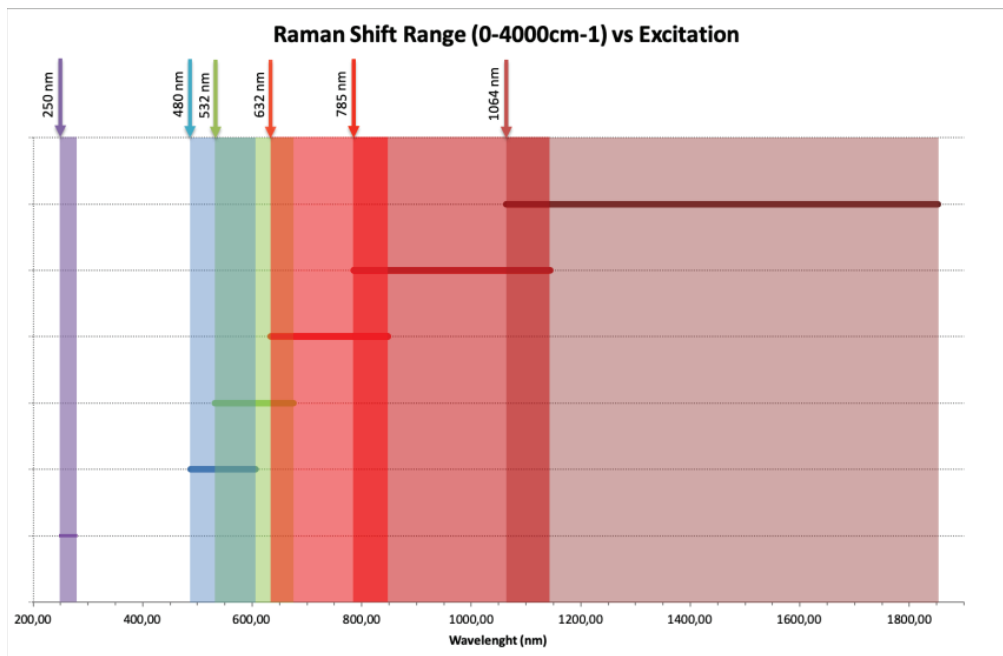

Figure 2 Raman shift range vs laser excitation. 


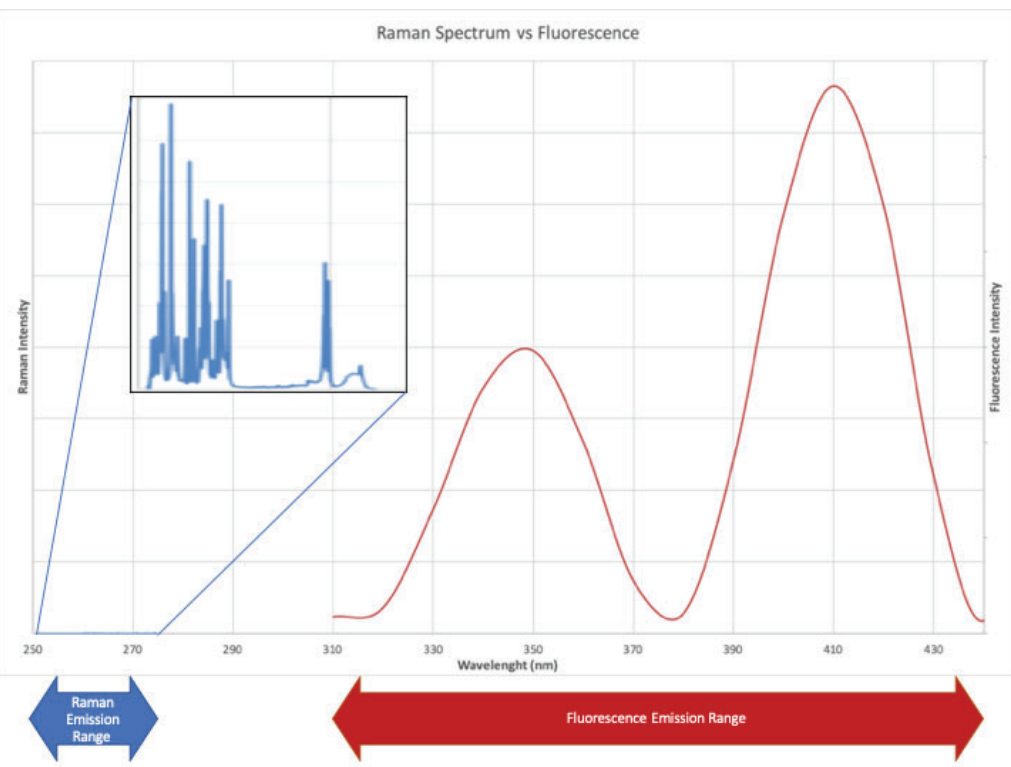

Figure 3 Raman and Fluorescence emission ranges for a $250 \mathrm{~nm}$ excitation.

\subsection{The instrument- Optical Payload}

As the Raman shift provides a weak signal compared to fluorescence effects, the instrument could have two different channels to assure success. The mission could include a Raman spectroscopy device but could also study atmosphere fluorescence. Both processes could be stimulated by using a single laser and the response signals could be analysed by a single spectrometer. Time resolved measurements should be included to differentiate the raised signal coming from the Raman shift or from the scattered fluorescence effect. In the case of the fluorescence signal, being of higher intensity, it could be used to acquire information in the tens of meters distance range across the atmosphere ${ }^{3}$. A similar approach is planned to be used in the OrganiCam instrument from Los Alamos National Laboratory, in a mission to Jupiter's moon Europa. The mission plans to use a combination of fluorescence/Raman instruments for a study of life ${ }^{25}$. Thus, authors suggest an instrument base line architecture as follows (see Figure 4):

1) The UV laser source will be separated using beam-splitters to transport the emitted light in free space into the different regions of study:

a. One of the beams is directed into a small probe side cavity (few $\mathrm{mm}$ ). Across the cavity (designed to stabilize the atmosphere) the device could perform transmission Raman. In the opposite side of the cavity, the receiving optics could bring the upcoming light into the spectrometer device. The instrument would be able to provide a more accurate study of single proximity molecules and possible amino acids.

b. The other beam could be directed to the probe side with an aperture opened to the Venusian atmosphere. The laser would illuminate, in this case, a few tens of meter into the atmosphere and later collect the fluorescence signal with a small telescope (around $100 \mathrm{~mm}$ aperture).

2) Both fluorescence and Raman signals, could be later joined by using a beam combiner element. The incoming light would then enter the spectrometer device that will independently analyse both signals. The Raman signal will be then used to study the molecules chemical bonds and possible functional groups, while the florescence signal will be focused on electronic structure to analyse aromatics, keynotes and aldehydes ${ }^{26}$.

The laser source and spectrometer instruments will have to be inserted into the protected core of the probe; guaranteeing that the sensitive instruments are kept away from the extreme environments. 


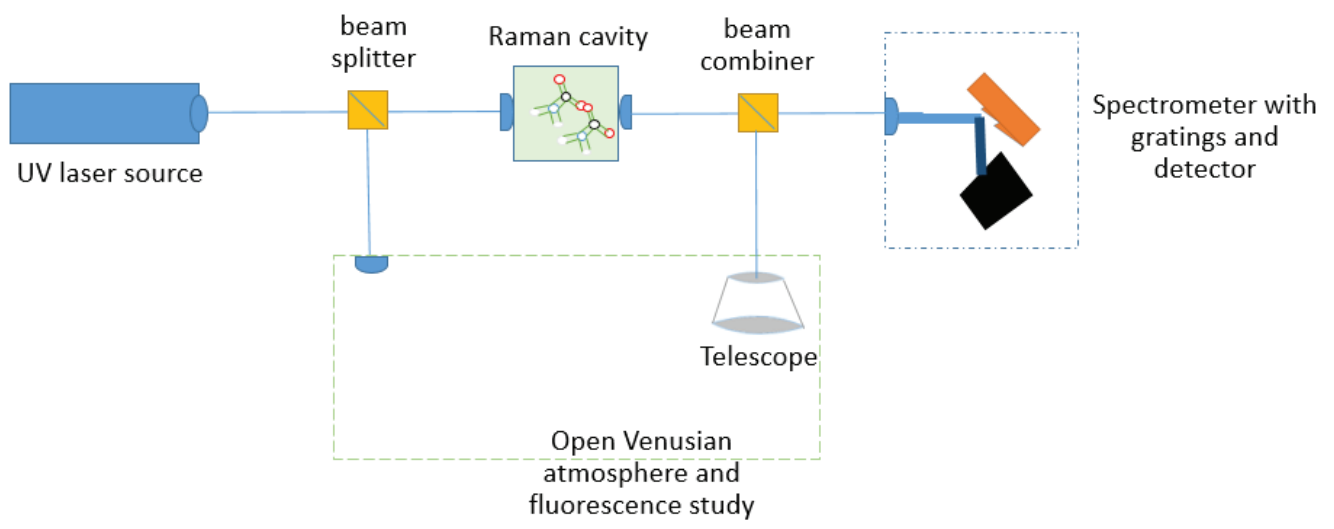

Figure 4 Schematic of the basic instrument base line. The laser could be obliquely placed near the receiving telescope.

\subsection{The laser excitation source}

Due to the Venus atmosphere thermal ranges and the interest in studying the presence of amino acids and their response to certain wavelengths, the proposed excitation source will be in the UV region, where these molecules show a great absorption and later fluorescence. UV lasers have recently been market accessible ${ }^{27}$. Even if nowadays reliable UV lasers can perform Raman spectroscopy in a lab environment, the laser qualification for being used in space missions is not yet a straight forward and standardized process ${ }^{29}$. However, examples of UV spectrometer laser devices, being used in space missions already exist, such as the SHERLOC instrument of the 2020 NASA mission ${ }^{15}$ or the UV-DPSS at the MOMA (Mars Organic Molecule Analyser) for the ExoMars 2022 mission ${ }^{28}$.

Different options could be available for the mission's purpose. However, the best solution will always be a trade-off between science capabilities (different sample analysis and used methods) and the mission constraints (budgets and environmental conditions). As a base-line, our preferred option due to the principal objective of studying amino acids, plus budget constraints and space heritage, we decided to propose a hollow cathode ion laser by a neon copper emitting at $248 \mathrm{~nm}$ wavelength ${ }^{30}$; similar as the one used for the SHERLOC instrument ${ }^{31}$.

Other UV configurations could be a slightly different UV laser emitter, changing the hollow cathode ion laser to a frequency doubled solid state laser, and even tuneable lasers with a wider emission wavelength ${ }^{32}$.

\subsection{Optics and analyzing setup}

The telescope implemented on the device acquiring the reflected signal from the Venusian atmosphere could have a baseline aperture around $100 \mathrm{~mm}$. UV light has the inconvenience of having a strong absorption when transmitted or reflected with optical components. Luckily, in recent years many research groups worked on $\mathrm{MgF}_{2}$ and $\mathrm{LiF}_{\mathrm{F}}$ coatings, which can provide UV and also far-UV (FUV) reflectance up to $80-90 \%$ (range around $270 \mathrm{~nm}$ ) ${ }^{33}$, making UV science feasible and efficient for such purposes. Similarly, the spectrometer gratings could be implemented using reflection holographic gratings, which are already available from companies such as Horiba.

Finally, we propose on doing a time-resolved Raman analysis ${ }^{3}$ by using an intensified charge-coupled device (ICCD) time gated measurement detector, to better separate the Raman and fluorescence signal.

\section{BALLOON AND GONDOLA CONSIDERATIONS}

Considering the altitude-of-interest region between 70 to $40 \mathrm{~km}$ and a pressure of $1 \mathrm{~atm}$, doing reverse engineering from earth helium balloons (balloon envelope and helium density of $0.18 \mathrm{Kg} / \mathrm{m}^{3}$ ), we choose a balloon with approximately a ratio of 0.25 of the mass of the gondola and payload. However, the system will have to include several balloon mechanisms (gas tanks, valves, vents and actuators) and a parachute (to reduce speed before the $70 \mathrm{~km}$ altitude point), which could represent higher constraints on the mission mass. A similar option was also planned for the ESA missions to Titan and Enceladus ${ }^{34}$. 
Assuming a slightly smaller payload to the one used in the Vega mission (7 Kg), and considering that the balloon system consisted of $15 \mathrm{Kg}$ mass for a balloon of $3.5 \mathrm{~m}$ diameter; we could target a gondola and payload mass below $5 \mathrm{Kg}$ that would require a balloon system, mechanisms and parachute of around $10 \mathrm{Kg}$.

\section{BUDGETS}

Considering previous Raman in-situ measurement instruments such as the ChemCam of about $6 \mathrm{Kg}$ (with three spectrometers), the SHERLOC with a $1.6 \mathrm{Kg}$ mass, and the ExoMars Raman with about $2.5 \mathrm{Kg}$, we could assume that the mission payload would be around $2 \mathrm{~kg}$. The Venusian instrument however will need additional instruments to work autonomously (batteries, computer), to communicate with an orbiter platform (radio antennas), to adjust the device to the thermal conditions (active/passive thermal regulation), and additional sensors to calculate position and perform additional studies (gyro, accelerometer, camera, pressure sensor, thermal sensor); these devices based on existing and space qualified Commercial off-the-shelf (COTS) components should be able to be fitted in a $2 \mathrm{Kg}$ mass envelope. Finally, we consider an additional $1 \mathrm{Kg}$ mass for providing a proper envelope for the harsh environmental conditions. This total amount of $5 \mathrm{Kg}$ would belong to the gondola section, while a final $10 \mathrm{~kg}$ mass will have to be added to the balloon and its subsystem components (Table 1).

In terms of power budgets we could assume $50 \mathrm{~W}$ for the optical payload, considering the NASA SHERLOC instrument ${ }^{35}$, and about $20 \mathrm{~W}$ for the rest of the required functional devices (communication, camera, thermal sensor, computer, etc.).

Table 1 Systems budgets

\begin{tabular}{|l|c|c|}
\hline System/Subsystem & Mass (Kg) & Power (W) \\
\hline $\begin{array}{l}\text { Main payload: } \\
\text { Raman/fluorescence instrument }\end{array}$ & 2 & 50 \\
\hline $\begin{array}{l}\text { Other devices: } \\
\begin{array}{l}\text { attitude and position } \\
\text { knowledge/cameras/pressure and } \\
\text { thermal sensor/batteries/etc. }\end{array}\end{array}$ & 2 & 10 \\
\hline $\begin{array}{l}\text { Envelope: } \\
\text { housing/thermal control }\end{array}$ & 1 & - \\
\hline $\begin{array}{l}\text { Balloon/deployment system: } \\
\text { balloon/mechanism/parachute }\end{array}$ & 10 & 70 \\
\hline TOTAL & 15 & \\
\hline
\end{tabular}

\section{CONCLUSION AND OUTLOOK}

In this article, we theorized and evaluated a draft design to send a small optical probe to study Venus's atmosphere. The science goals and approximate required budgets have been presented showing that the mission could be realizable with relatively low budgets. Further steps should be a detailed evaluation and qualification of designs to assure that the instruments can survive the harsh Venusian conditions (housing study and thermal analysis of devices in operation). Moreover, authors also suggest a European effort to qualify standard laser and laser Raman devices able to be sent to different space bodies, since laser qualification campaigns could represent by itself a mission constraint. Nowadays, the devices and mechanisms required for the correct functioning of the probe are market accessible by COTS components, and in most cases, already qualified for space missions.

The need for sending similar probes to Venus was already proposed by the Venus Science and Technology Definition Team back in $2009^{36}$, where spectrometer devices mounted in descending balloons were thought to be a good solution to explore the planet's atmosphere. Here a more detailed optical baseline design of how this device could be sketched has been shown. If budgets allow it, other devices could be included in the descending probe such as a nephelometer; or other scientific measurement devices of interest. 


\section{ACKNOWLEDGEMENTS}

Authors want to thanks the Dr. Rossá Mac Ciarnáin from Physicsproof.com for the proofreading service.

\section{REFERENCES}

[1] Otroshchenko, V.A., Surkov, Y. A., "The possibility of organic molecule formation in the Venus atmosphere, Origins of life," Springer Exobiology, Vol. 5, pp. 487-490 (1974). Doi: 10.1007/BF01207647

[2] Taylor, F. W., Svedhem, H., Head J. W., "Venus: The Atmosphere, Climate, Surface, Interior and Near-Space Environment of an Earth-Like Planet," Space Science Reviews Vol. 214(35) (2018). Doi:10.1007/s11214-0180467-8

[3] Michael, S.A., et. al., "Remote Raman Spectroscopy for Planetary Exploration: A Review," Applied Spectroscopy, Vol. 66(2) (2012). Doi: 10.1366/11-06535

[4] Frandsen, B. N., Wennberg, P. O., Kjaergaard, H. G., "Identification of OSSO as a near-UV absorber in the Venusian atmosphere, Geophysical Research Letters," Vol. 43(21) (2016). Doi: 10.1002/2016GL070916

[5] Limaye, S. S., Mogul, R., Smith, D. J., Ansari, A. H., Słowik, G. P., Vaishampayan, P., "Venus' Spectral Signatures and the Potential for Life in the Clouds," Astrobiology Vol. 18 (2018). Doi: 10.1089/ast.2017.1783.

[6] Web link: https://www.esa.int/About_Us/ESA_Permanent_Mission_in_Russia/Venera-D. Accessed on the $18 / 12 / 2020$.

[7] Report of the Venera-D Join Science Definition Team. Venera-D: Expanding our Horizon of terrestrial Planet Climate and Geology through the Comprehensive Exploration of Venus. (2017). Web link: https://www.lpi.usra.edu/vexag/reports/Venera-D-STDT013117.pdf. Accessed on the 18/12/2020.

[8] Web link: https://www.nasa.gov/feature/goddard/2017/nasa-studies-cubesat-mission-to-solve-venusianmystery. Accessed on the 18/12/2020.

[9] Web link: https:/www.nasa.gov/feature/goddard/2020/nasa-goddard-team-selected-to-design-concept-forprobe-of-mysterious-venus-atmosphere. Accessed on the 13/05/2020.

[10] Web link: https://nssdc.gsfc.nasa.gov/nmc/spacecraft/display.action?id=1967-058A Accessed on the 13/05/2020.

[11] Web link: https://stardust.jpl.nasa.gov/comets/vega.html. Accessed on the 13/05/2020.

[12] Web link: https://nssdc.gsfc.nasa.gov/nmc/spacecraft/display.action?id=1984-128F. Accessed on the $15 / 05 / 2020$.

[13] Web link: https://solarsystem.nasa.gov/missions/pioneer-venus-2/in-depth/. Accessed on the 13/05/2020.

[14] Misra, A. K., et al., "Remote Raman Spectroscopic Detection of Minerals and Organics Under Illuminated Conditions from a Distance of $10 \mathrm{~m}$ Using a Single $532 \mathrm{~nm}$ Laser Pulse," Applied Spectroscopy, Vol. 60 (2) (2006).

[15]Beegle, L., Bhartia, R., White, M., DeFlores, L., Abbey, W., Wu, Y.H., Cameron, B., Moore, J., Fries, M., Burton, A., et al., "Sherloc: Scanning habitable environments with Raman and luminescence for organics and chemicals," In Proceedings of the IEEE Aerospace Conference, Big Sky, MT, USA, pp. 1-11 (2015).

[16] Ribes-Pleguezuelo, P., Guilhot, D., Gilaberte Basset, M., Beckert, E., Eberhardt, R., Tünnermann, A., "Insights of the Qualified ExoMars Laser and Mechanical Considerations of Its Assembly Process. MDPI Instruments," Vol. 3(25) (2019).

[17] Sharma, S. K., et al., "Remote-Raman spectroscopic study of minerals under supercritical CO2 relevant Venus exploration," Elsvier Molecular and Bimolecular Spectroscopy, Vol. 80(1), pp. 75-81 (2011). Doi: 10.1016/j.saa.2011.01.033

[18] Web

link: https://www.esa.int/Science_Exploration/Space_Science/Venus_Express/The_unexpected_temperature_profile_ of_Venus_s_atmosphere. Accessed on the 13/05/2020.

[19] Taylor, F.W., et al., "Venus: The Atmosphere, Climate, Surface, Interior and Near-Space Environment of an Earth-Like Planet, Springer Space Science Reviews," Vol. 214, pp. 35 (2018). Doi. 10.1007/s11214-018-04678.

[20] Wilson, C.F., "Venus: Key to understanding the evolution of terrestrial planets," White paper: L2/L3 Missions in the ESA science programme (2013). 
[21] Wilson, C.F., et al., “The 2010 European Venus Explorer (EVE) mission proposal,” Experimental Astronomy (2013). Doi: 10.1007/s10686-011-9259-9.

[22] Image from

weblink: https://www.esa.int/Science_Exploration/Space_Science/Venus_Express/The_unexpected_temperature_profile_ of_Venus_s_atmosphere. Accessed on the 02/12/2020.

[23] Seager, S., et. al., "The Venusian Lower Atmosphere Haze as a Depot for Desiccated Microbial Life: A Proposed Life Cycle for Persistence of the Venusian Aerial Biosphere," Astrobiology, Ahead of print. Doi: 10.1089/ast.2020.2244.

[24] Greaves, J. S., et. al., "Phosphine gas in the cloud decks of Venus," Nat. Astron. (2020). Doi: 10.1038/s41550020-1174-4

[25] Web link: https://www.lanl.gov/discover/publications/connections/2018/2018-12/science.php. Accessed on the 04/12/2020.

[26]Hug, W.F., et al., "Deep UV Raman and fluorescence spectroscopy for real-time in-situ process monitoring," Proc. SPIE, Next-Generation Spectroscopic Technologies XIII, Vol. 11390, pp. 113900X (2020). Doi: $10.1117 / 12.2556653$

[27] Web link: https://www.rp-photonics.com/ultraviolet_lasers.html. Accessed on the 09/04/2020.

[28] Wessels, P., et al., "UV-DPSS Laser Flight Model for the MOMA Instrument of the ExoMars 2020 Mission," Proc. SPIE, Vol. 11180, pp. 111801B (2019). Doi: 10.1117/12.2535966

[29] Guilhot, D., et al., "Laser Technology in Photonic application for Space," MDPI Instruments, Vol. 3(3) (2019). Doi: 10.3390/instruments3030050.

[30] Storrie-Lombardi M. C., et. al., "Hollow cathode ion lasers for deep ultraviolet Raman spectroscopy and fluorescence imaging," Review of Scientific Instruments, Vol. 72(12) (2001).

[31] Photonsystems-Space Qualified Deep UV. Available online: https://photonsystems.com/space-qualified/ . Accessed on the 16/07/2020.

[32]Fahey, M., et al., "Ultraviolet Laser Development for Planetary Lander Missions," 2020 IEEE Aerospace Conference (2020). Doi: 10.1109/AERO47225.2020.9172711

[33] Ribes-Pleguezuelo, et. al., "UV astronomy with small satellites," Preprint: Astrophysics, Instrumentation and Methods for Astrophysics (2020).

[34] Coustenis, A., Atreya, S.K., Balint, T. et al. "TandEM: Titan and Enceladus mission,” Exp Astron Vol. 23, pp. 893-946 (2009). Doi: 10.1007/s10686-008-9103-z

[35] Web link. NASA. https://mars.nasa.gov/mars2020/spacecraft/instruments/sherloc/. Accessed on the 15/09/2020.

[36] Bullock, M. A., et al., "A Venus Flagship Mission: Report of the Venus Science and Technology Definition Team," 40th Lunar and Planetary Science Conference (2009). 